\title{
Research on Applications of Fuzzy Self-adaptive PID Control in Synchronous Control System Based on Electro-Hydraulic Proportional Technology
}

\author{
Jinsong Chen \\ Institute of Physics and Electronic Engineering, Yibin University, Yibin City, Sichuan \\ Province, 644000
}

hunter2011@foxmail.com

\begin{abstract}
Keywords: Synchronous control system, Electro-hydraulic proportional, Fuzzy self-adaptive PID control
\end{abstract}

\begin{abstract}
Electro-hydraulic proportional (EHP) control system is widely applied in the engineering field because of the characteristics of its simple structure, high precision, easy to auto control and applied to high-power. This paper describes the principle of fuzzy self-adaptive PID control, and then applies the fuzzy self-adaptive PID control in synchronous control system based on electro-hydraulic proportional technology, finally gives the simulation results which are consistent with expectation in order to provide some references to the related researchers.
\end{abstract}

\section{Introduction}

The traditional electro hydraulic servo valve has a very strict requirement on the cleanliness of the fluid medium. At the same time, the manufacturing cost and maintenance cost are relatively high. The system energy consumption is relatively large. Electro hydraulic proportional control arises at the historic moment, which is characterized by its low cost, energy saving, convenient maintenance and adaptation to high power control, which is widely used in modern industry. Hydraulic actuators are usually hydraulic cylinder or hydraulic motor, the output parameters can only be displacement, velocity, acceleration and force, or angle, angular velocity, angular acceleration and torque. The system can improve the accuracy and dynamic quality of the system by setting up the feedback loop of the intermediate variables of the hydraulic pressure and flow rate and the mechanical parameters of the system. The signal processing unit can be realized by using analog electronic circuit, digital micro processing chip or microcomputer. The digital integrated circuit has the advantages of accuracy, reliability and stability.

\section{Principle of Fuzzy Self-adaptive PID Control}

For electro hydraulic proportional system, the fixed control parameters cannot match with the time variant characteristics due to the time varying characteristics of the object. Therefore, the actual situation of the scene requires that the PID parameters can be adjusted automatically. The experience knowledge that is accumulated in the long time of the operation is the model of the control rule, and then the optimal adjustment of PID parameters is realized. However, due to the experience of the operator is not easy to accurately describe, the control process of various signal quantity and evaluation index is not easy to say, the introduction of fuzzy theory is the effective way to solve this problem. Using the basic theory and method of fuzzy mathematics, the conditions of the rules, the action of fuzzy sets, and the fuzzy control rules and the relevant information as knowledge into the knowledge base of the motion controller, and then the main controller according to the actual response of the control system, the use of fuzzy reasoning, can automatically achieve the optimal adjustment of PID parameters, which is the fuzzy adaptive PID control. The fuzzy adaptive PID controller is used as the input of the error and the error, and the PID parameters are modified online by using the fuzzy control rules. The difference between it and the conventional fuzzy control is that it can meet the requirements of the PID parameter self-adaptive at different time, and realize the complex control of the fuzzy and the traditional PID controller. 
$\mathrm{P} 1 \sim \mathrm{P} 2\left(\mathrm{e}>0, \mathrm{e}_{\mathrm{c}}<0\right)$ : the system presents the trend of steady change, so at the beginning, the larger proportion coefficient is to speed up the system response speed, and the smaller integral coefficient and differential coefficient. When the speed is close to the P2 point, it should reduce the proportion coefficient and even eliminate the integral effect.

P2 P3 $\left(\mathrm{e}<0, \mathrm{e}_{\mathrm{c}}<0\right)$ : the output value of the system is more than the steady state value, and continues to change the direction of the deviation. At this stage, the control function should be tried to depress the overshoot. During the whole stage, the large differential coefficient should be taken, while the proportion coefficient and integral coefficient are smaller.

P3 P4 (e<0, $\left.e_{c}>0\right)$ : the speed of system output tends to be stable, which should be eliminated as soon as possible. When the system enters the steady state, the $\mathrm{C}$ points should be used to reduce the overshoot. The differential coefficient should be increased.

P4 P5 $\left(\mathrm{e}>0, \mathrm{e}_{\mathrm{c}}>0\right)$ : the system has a high profile, the deviation direction changes, at this point, the $\mathrm{P} 4$ points should be taken to a large differential coefficient, when the P5 point is close to the appropriate differential coefficient, increase the integral coefficient and the proportion coefficient.

The whole set of fuzzy rules are given. The expressions of the phase plane can be more helpful to achieve the relationship between E and EC.

\section{Applications of Fuzzy Self-adaptive PID Control in Synchronous Control System}

Simulation Model. We design the fuzzy controller in the double cylinder respectively, and the right cylinder as the following cylinder, the input signal is the difference between the position error of the double cylinder synchronous position and the position feedback of the loop. The double cylinder loop is based on the error and error rate of the fuzzy controller to get the output control. Based on the simulation model of electro hydraulic proportional synchronous control system, we use SIMULINK to construct a double cylinder synchronous simulation model under the conventional fuzzy controller, as shown in Figure 1.

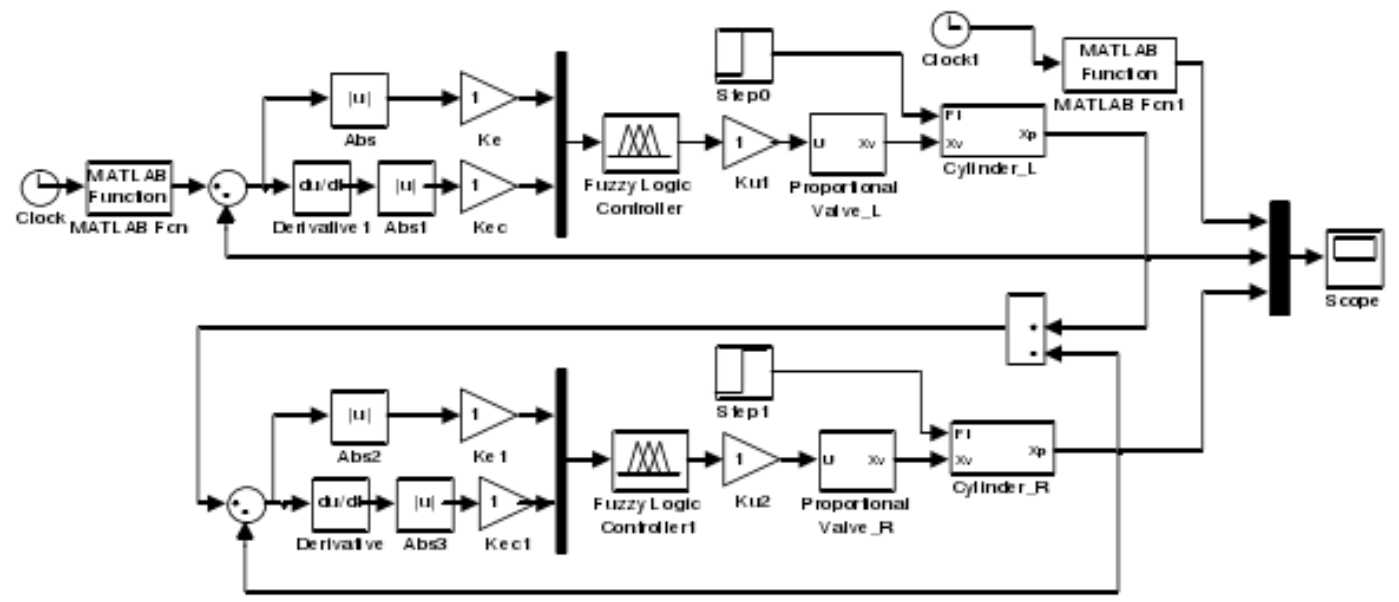

Figure 1. Simulation Model of Fuzzy Controller

Parameter Selection. Fuzzy self-adaptive PID controller rule table is the same as the conventional fuzzy controller. The input variables of the controller, the error e and its change rate $\mathrm{e}_{c}$, the output variable is PID parameter are shown in the following table.

Table 1. System Program Results (Threshold=0.8)

\begin{tabular}{|l|l|l|l|l|l|}
\hline variable & $\mathrm{e}$ & $\mathrm{e}_{\mathrm{c}}$ & $\mathrm{k}_{\mathrm{p}}$ & $\mathrm{k}_{\mathrm{i}}$ & $\mathrm{k}_{\mathrm{d}}$ \\
\hline language varible & $\mathrm{E}$ & $\mathrm{EC}$ & $\mathrm{k}_{\mathrm{p}}$ & $\mathrm{k}_{\mathrm{i}}$ & $\mathrm{k}_{\mathrm{d}}$ \\
\hline basic domain & \multicolumn{3}{|c|}{$[-2,2]$} & \multicolumn{5}{|c|}{$[0,1]$} \\
\hline fuzzy subgruop & \multicolumn{2}{|c|}{$[\mathrm{NB}, \mathrm{NM}, \mathrm{NS}, \mathrm{ZE}, \mathrm{PS}, \mathrm{PM}, \mathrm{PB}]$} & \multicolumn{2}{|c|}{$[$ ZE, PS, PB] } \\
\hline fuzzy domain & {$[-6,6]$} & & & $6,6]$ & 40 \\
\hline quantification factor & 6 & 60 & 6 & 6 & \\
\hline
\end{tabular}


Two Sub-modules. Figure 2 and Figure 3 respectively give the systems of fuzzy control module and self-adaptive PID module.

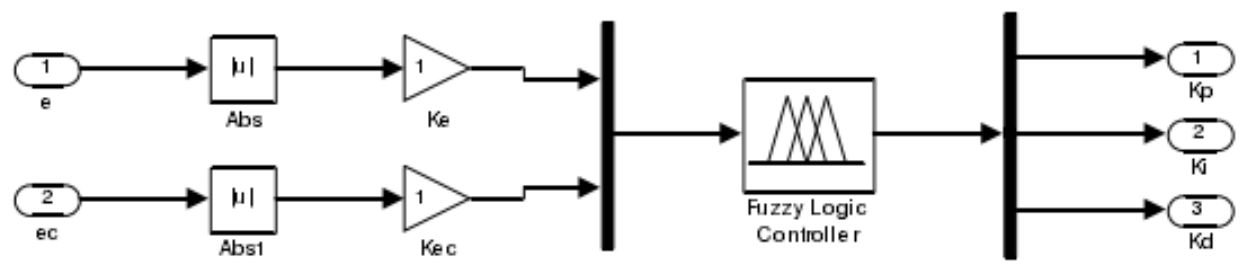

Fig. 2. System of of Fuzzy Controller

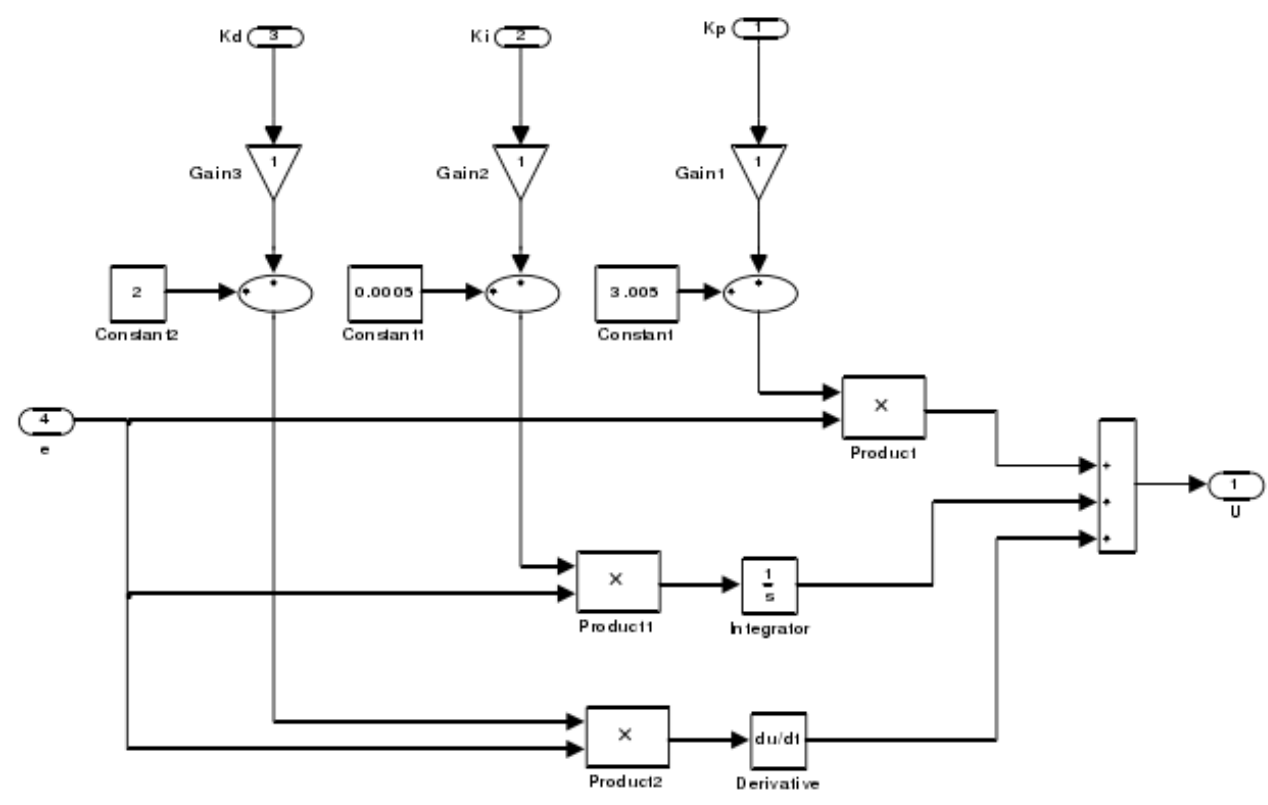

Fig. 3. System of of Self-adaptive PID

Simulation Results. We design fuzzy control module based on FIS Matlab. The input variables are $\mathrm{e}, \mathrm{e}_{\mathrm{c}}$, and the output variables are $\mathrm{K}_{\mathrm{p}}, \mathrm{K}_{\mathrm{i}}$ and $\mathrm{K}_{\mathrm{d}}$. The simulation curves are shown in figure 4.25. With the PID control, fuzzy control under single loop step response of phase contrast. The fuzzy adaptive PID control has better control performance compared with fuzzy control and control, and the response speed of the system is the fastest, with $\mathrm{rt}=15 \mathrm{~ms}$, and the regulation time $=55 \mathrm{~ms}$.

Fuzzy control system cannot be established accurately, through expert experience, the fuzzy control rules and the method of system control can be realized. PID control, fuzzy control and fuzzy adaptive PID control the unit step response of the system. It can be seen that the fuzzy control can make the response speed of the system change fast, but it is inevitable to produce steady state error, and the control effect of fuzzy control depends on the establishment of control rules. The fuzzy adaptive PID control can make the system overshoot and reduce or even have no overshoot, adjust time to shorten. Because of its strong adaptability to the parameters of the object, it is suitable for the control of the electro-hydraulic proportional control of the system. From the simulation results, when the PID parameters in the dual cylinder controller can be adjusted according to the fuzzy rules, the control effect of the two cylinders is improved obviously. For example, when the load is the same, the maximum tracking error of PID is $1.1 \mathrm{~mm}$, the maximum synchronization error is $0.09 \mathrm{~mm}$, and the maximum tracking error is $0.06 \mathrm{~mm}$, and the maximum synchronization error is $0.026 \mathrm{~mm}$. Obviously, the fuzzy adaptive PID control improves the following characteristics of the cylinder, and the simultaneous error of the two cylinders can achieve more satisfactory results. But because the simulation is finished in the ideal environment, the algorithm design is only for the control of the system to find a good control scheme. The actual system's response and synchronization error can meet the requirements in the actual working condition. 


\section{Conclusion}

With the wide application of hydraulic technology in engineering field, the synchronous motion of hydraulic mechanism is one of the key techniques to study the hydraulic equipment with synchronous requirements. It has important research value to solve the problem of synchronization in hydraulic equipment. This paper uses the Matlab/SIMULINK software to establish the corresponding simulation model and achieve the expected simulation results which may has significant influence on prompting synchronous control system based on EHP technology.

\section{Acknowledgements}

Project: Study on fuzzy PID control of electro hydraulic proportional system, Number: 2011ZGY002

\section{References}

[1] Y.J. Xing, Research of Synchronous Control System Based on Electro-Hydraulic Proportional Technology. Nanjing University of Aeronautics and Astronautics, 2010

[2] Y.X. Yang, D.L. Cui, A.J. Zhou, Self -adaptive Fuzzy PID Controller and Realizing the Control System in Simulink Environment, Ship Electronic Engineering, 2010(4):127-130

[3] L.P. Zhang, L.X. Ma, Z.Z. Jin, Design of Fuzzy Self-adaptive PID Control System for Resistance Furnace Temperature, Material \& Heat Treatment, 2012(7):234-239.

[4] R.L. He, Study on the Speed Regulation System of Permanent Magnetic Synchronization Motor Based on Fuzzy Adaptive PID Controller. Chang'an University, 2014 